\title{
Granulomatosis linfomatoide: una entidad poco conocida
}

\author{
J. D. Cumplido Burón, I. Blancas López-Barajas, J. A. García García, N. Cárdenas Quesada, P. Iglesias Rozas,
} M. J. Sánchez García, T. Delgado Ureña, B. Ríos Pozo, J. L. García-Puche

\section{Resumen}

Propósito: describir un caso clínico de granulomatosis linfomatoide, y realizar una revisión de la bibliografía existente para conocer mejor dicha enfermedad.

Material y métodos: varón de 27 años con antecedentes de linfoma no Hodgkin B a los 17 años que debuta con insuficiencia respiratoria tras diez años en revisiones periódicas.

Conclusiones: la granulomatosis linfomatoide constituye una entidad rara, de mal pronóstico y en la que no se ha definido el mejor tratamiento posible, existiendo diversas posibilidades y siendo necesario un mayor estudio de dicha entidad con el fin de mejorar su pronóstico.

Palabras clave: Granulomatosis linfomatoide. Virus Epstein Barr. Linfoma.

Oncología, 2007; 30 (1):21-24

\section{Summary}

Purpose: To describe a clinical case of lymphomatoid granulomatosis and to make a review of the existing bibliography for the better knowing of the disease.

Material and methods: A 27 year old man with a history of B-cell lymphoma at he age of 17 years that started with respiratory insufficiency after ten years of periodic checkups.

Conclusion: Lymphomatoid granulomatosis is a rare entity, with a poor prognosis and a lack of an established treatment. Although different treatments have been applied, further studies are necessary in order to improve its prognosis.

Key words: Lymphomatoid granulomatosis. Epstein. Barr virus. Lymphoma. 


\section{Introducción}

La granulomatosis linfomatoide es una entidad infrecuente, desconocida por gran cantidad de clínicos y cuyo tratamiento y pronóstico no está definido. Se trata de un proceso linfoproliferativo angiocéntrico y angiodestructivo que afecta principalmente regiones extranodales. Plantea un diagnóstico complicado dada su similitud con procesos vasculíticos por lo que es importante la sospecha clínica. Actualmente se considera un proceso de células B relacionado con infección por el virus de Ebstein Barr y con linfoma B de células grandes. Presentamos el caso de un paciente afecto de dicha enfermedad con afectación pulmonar y a nivel del sistema nervioso central, además de realizar una revisión de la literatura existente para conocer las posibilidades terapéuticas y la evolución de dicho cuadro.

\section{Caso clínico}

Paciente varón de 27 años con antecedentes de hepatitis tipo A en la infancia e intervención de sinus pilonidal a los 20 años, sin antecedentes familiares de interés. Como antecedente personal, en diciembre de 1996 debuta con disartria y parestesias en hemicuerpo derecho, por lo que se inicia estudio diagnóstico detectándose en una TAC craneal la presencia de imágenes nodulares múltiples. Se procedió a toma de biopsia de una lesión frontal, cuyo resultado histopatológico reveló el diagnóstico de linfoma no Hodgkin de células B de células pequeñas. Entre enero y marzo de 1997 recibe radioterapia holocraneal (a dosis de 46 Gy) junto con quimioterapia intratecal con metotrexate, con respuesta clínica y estabilización radiológica, por lo que se decide administración de quimioterapia sistémica en régimen PROMACE (x2)- MOOP (x2) (ciclofosfamida, doxorrubicina, etopósido, metotrexate, vincristina y procarbacina). Con dicho tratamiento el paciente alcanza respuesta completa radiológica con persistencia de lesiones residuales posteriores al tratamiento, por lo que en octubre de 1997 se deciden revisiones periódicas. El paciente continuó dichas revisiones hasta diciembre de 2005 sin objetivarse progresión de su enfermedad.

En este intervalo, únicamente hay que destacar un cuadro radiológico detectado en febrero de 2004, al detectarse en radiografía simple de tórax la presencia de infiltrados pulmonares bilaterales, sin sintomatología acompañante, que fue valorado por parte del Servicio de Neumología como un cuadro viral, dado su resolución espontánea en el plazo de ocho semanas.

El paciente debuta en febrero de 2006 con disnea de mínimos esfuerzos, motivo por el que acude al Servicio de Urgencias, objetivándose una hipoxemia importante secundaria a afectación pulmonar por infiltrados pulmonares bilaterales. Se procede a su ingreso en el Servicio de Respiratorio, y se procede a estudio diagnóstico. Se realiza una fibrobroncoscopia con lavado bronquial y toma de biopsias, observándose únicamente una linfocitosis en el lavado bronquial, sin otros hallazgos. La evolución del paciente tras la instauración de tratamiento corticoideo fue satisfactoria por lo que fue dado de alta. Siete días más tarde, es ingresado nuevamente por un cuadro clínico de similares características, con disnea de reposo, por lo que se decide realizar una biopsia pulmonar por parte del servicio de Cirugía Torácica.

Previo a dicha toma de biopsia, el paciente presenta dos convulsiones tónico-clónicas generalizadas por lo que se solicita una TAC craneal, donde se observa una lesión frontal izquierda con edema asociado, de nueva aparición.

Finalmente se realiza la biopsia pulmonar, con resultado anatomopatológico de granulomatosis linfomatoide grado I. En el estudio de extensión realizado, se comprueba infiltración de médula ósea secundaria a granulomatosis linfomatoide. Serología negativa salvo positividad para IgG VEB. TACbody: infiltrados pulmonares bilaterales, esplenomegalia y la lesión frontal ya descrita. Ante el diagnóstico de granulomatosis linfomatoide sistémica (afectación pulmonar, cerebral y médula ósea), se decide en sesión clínica inicio de tratamiento quimioterápico en régimen CHOP-rituximab (ciclofosfamida, adriamicina, vincristina, prednisona). Tras dos ciclos de dicho tratamiento, el paciente no presenta respuesta de ningún tipo a la quimioterapia, ya sea clínica o radiológica, a lo que se añadió las complicaciones secundarias del tratamiento quimioterápico con una pancitopenia severa, llevando a un deterioro progresivo del paciente en su estado general y un empeoramiento de su función respiratoria, sin responder al tratamiento instaurado (antibiótico, 

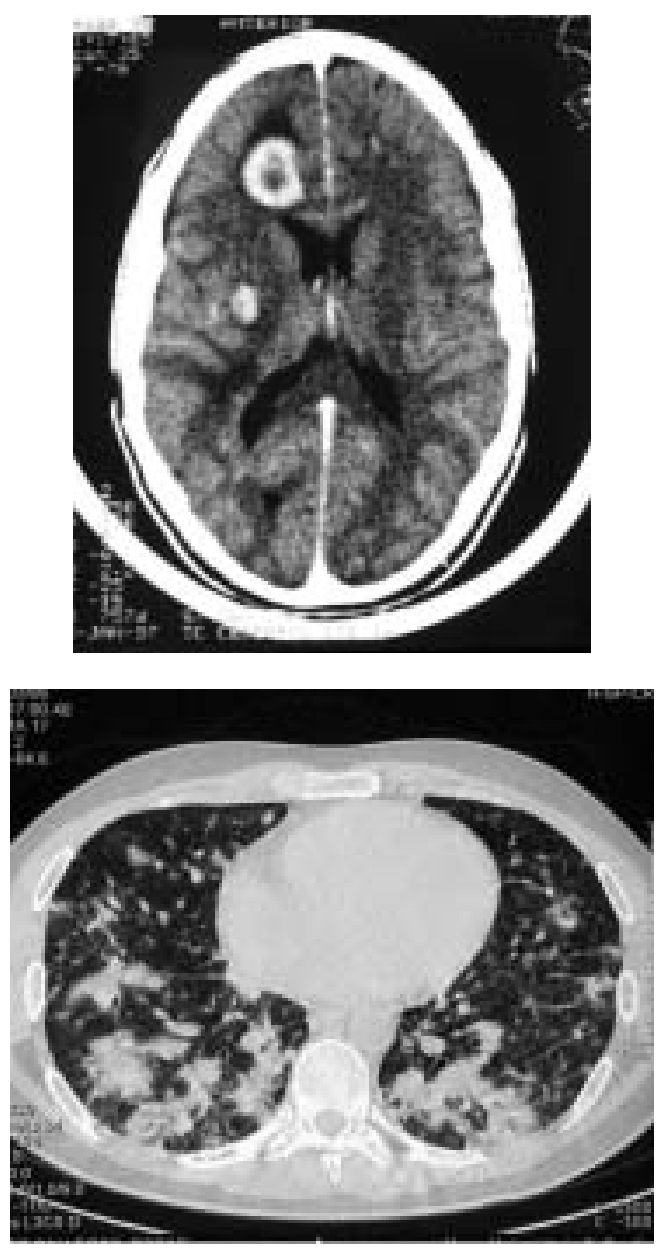

factor estimulante de colonias, oxígeno hiperbárico), y produciéndose el éxitus cuatro meses tras el diagnóstico.

\section{Discusión}

La granulomatosis linfomatoide se define como un proceso linfoproliferativo angiocéntrico y angiodestructivo que afecta "lugares extranodales" y que se asocial con frecuencia al virus de Epstein-Barr. Fue descrita por primera vez en 1972, por Liebow y cols $^{1}$, destacando como una característica la afectación de "tejido linfático" extranodal, con especial afectación a nivel pulmonar, en cerca de un $50 \%$ de los casos, afectando también a piel, SNC, tracto GI, hígado, bazo, riñones y corazón En nuestro caso podemos observar como el órgano principalmente afectado, así como causante de la sintomatología es el pulmón. No hay que olvidar la esplenomegalia que presenta el paciente, así como la afectación cerebral, lugares comúnmente afectados por esta entidad. Plantea diagnóstico diferencial con procesos vasculíticos (granulomatosis de Wegener principalmente) existiendo abundante bibliografía al respecto $^{2}$, dada la afectación multiorgánica característica así como las características histológicas de afectación en los vasos. Con esta similitud, el diagnóstico diferencial es obligado y en el caso que presentamos, la demora diagnóstica está en parte debida a esta similitud. A la hora de realizar el diagnóstico diferencial, habrá que solicitar valoración de anticuerpos antinucleares (en nuestro caso, negativos), así como examen histológica de lesiones accesibles. En nuestro caso, tras la biopsia a través de toracotomía se llegó al diagnóstico anatomopatológico, además de confirmar positividad para células de Ebstein-Barr. Hay que tener en cuenta que llegar a este diagnóstico histológico es complicado, dada la similitud con las vasculitis, por lo que se están realizando en los últimos años estudios mediante citometría y PCR (reacción en cadena para polimerasa) que ayudan al diagnóstico de dicha entidad al detectar VEB, o un fenotipo característico de células $\mathrm{T}$ reactivas $^{3,4}$.

Se trata de una entidad rara, infrecuente, y cuyo conocimiento es escaso. Su incidencia es mayor entre adultos jóvenes varones, algo que ocurre en nuestro caso. Llegado al diagnóstico definitivo, realizamos sesión clínica conjunta con el Servicio de Hematología y de Anatomía Patológica, revisando la bibliografía existente y las posibilidades terapéuticas. El abanico terapéutico descrito en la literatura es amplio. Existen actitudes que varían entre la espera terapéutica para pacientes asintomáticos, donde se ha llegado a describir casos de remisión espontánea ${ }^{5}$ hasta tratamiento con quimioterapia a altas dosis seguido autotrasplante de médula ósea. Se ha comunicado el caso de un paciente tratado con autotrasplante de médula e interferón alfa- 2 a de mantenimiento que se mantiene libre de enfermedad 8 años tras el trasplante ${ }^{6}$.

En la literatura podemos encontrar tratamiento con ciclofosfamida junto corticoides en pacientes afectos de granulomatosis linfomatoide, con afectación cerebral, pulmonar o cutánea, alcanzando supervivencias entre los 2 y 24 meses $^{2,6,7}$. También se ha descrito el empleo de pautas consideradas están- 
dar en el tratamiento de linfomas no Hodgkin de células $\mathrm{B}$, como $\mathrm{CHOP}$ en pacientes con estas mismas características. Oliveras y cols ${ }^{8}$ describen este tipo de terapia combinada con prednisona en una mujer de 67 años, que permanece viva a los 10 meses tras finalizar el tratamiento. En este mismo sentido, Petrella y cols ${ }^{9}$ describen un caso únicamente de un varón de 33 años con afectación pulmonar, que tras la administración de CHOP y radioterapia de consolidación permanece vivo a los 13 meses de finalizar el tratamiento. Pero los agentes citotóxicos no son la única arma terapéutica descrita en el tratamiento de esta patología. Existen trabajos en los que se emplean un tratamiento inmunomodulador con interferón alfa- $2 \mathrm{~b}$, en pacientes con infección por VEB activa, basándose en el efecto inmunomodulador, antiviral y antiproliferativo del interferón. Con este tratamiento se han descrito respuestas del $100 \%$, eso sí, en una serie de 4 pacientes, 3 alcanzando una respuesta completa y permaneciendo libres de enfermedad tras 60 meses de seguimiento ${ }^{10}$.

Debido al avance en los últimos años en el campo de los anticuerpos monoclonales y el conocido efecto del rituximab en neoplasias hematológicas, no faltan los estudios en los que se estudia la eficacia del rituximab (anticuerpo anti-CD20) debido a su consideración como un linfoma de células B. Los resultados obtenidos son contradictorios, por lo que son necesarios un mayor número de estudios para extraer conclusiones ${ }^{11-13}$.

En definitiva, la granulomatosis linfomatoide constituye una entidad rara, de mal pronóstico y en la que no se ha definido el mejor tratamiento posible, existiendo diversas posibilidades y siendo necesario un mayor estudio de dicha entidad con el fin de mejorar su pronóstico.

\section{Bibliografía}

1. Liebow AA, Carrington CR, Friedman PJ. Lymphomatoid granulomatosis. Hum Pathol 1972; 3: 457-558.

2. Wu S, Min Y, Ostrzega $\mathrm{N}$ et al. Lymphomatoid granulomatosis: A rare mimicker of vasculitis. J Rheumatol 2005; 32: 2242-5.

3. Katzenstein AL, Peiper SC. Detection of Epstein-Barr virus genomes in lymphomatoid granulomatosis: analysis of 29 cases by the polymerase chain reaction technique. Mod Pathol 1990; 3:435-41.

4. Taniere P, Thivolet-Bejui F, Vitrey D et al. Lymphomatoid granulomatosis- a report of four cases: evidence for B phenotype of the tumoral cells. Eur Respir J 1998; 12 (1): 102 6.

5. Katzenstein AL, Carrington CB, Liebow AA. Lymphomatoid granulomatosis: prospective clinical and therapeutic experience over 10 years. Cancer 1979; 43: 360-73.

6. Johnston A, Coyle L, Nevell D. Prolonged remission of refractory lymphomatoid granulomatosis after hemopoietic stem cell transplantation with post-transplantation maintenance interferon. Leuk Lymphoma 2006; 47 (2): 323-8.

7. Kerslake R, Rowe D, Worthington BS. CT and MR imaging of CNS lymphomatoid granulomatosis. Neuroradiology 1991; 33(3): 269-71.

8. Patton WF, Lynch III JP. Lymphomatoid granulomatosis. Clinicopathologic study of four cases and literature review. Medicine 1982; 61 (1): 1-12.

9. Petrella TM, Walter IR, Jones GW et al. Radiotherapy to control CNS lymphomatoid granulomatosis: a case report and review of the literature. Am J Hematol 1999;62 (4):239-41.

10. Wilson WH, Kingma DW, Raffeld M et al. Association of lymphomatoid granulomatosis with Epstein-Barr viral infection of B lymphocytes and response to interferon-alpha 2b. Blood 1996; 87(11): 4531-7.

11. Polizzotto MN, Dawson MA, Opat SS. Failure of rituximab monotherapy in lymphomatoid granulomatosis. Eur J Haematol 2005; 73 (3): 273-80.

12. Jordan K, Grothey A, Grothe W et al. Successful treatment of mediastinal lymphomatoid granulomatosis with rituximab monotherapy. Eur J Haematol 2005; 74 (3): 263-6.

13. Zaidi A, Kampalath B, Peltier WL et al. Successful treatment of systemic and central nervous system lymphomatoid granulomatosis with rituximab. Leuk Lymphoma 2004; 45 (4): 777-80.
Correspondencia:

Dr. J. D. Cumplido Burón Unidad Clínica de Oncología Hospital Clínico San Cecilio C/ Dr. Olóriz, s/n E-18003 Granada davidcumplido@hotmail.com 